Vol 11, Issue 10, 2018

\title{
STANDARDIZATION OF SNOWDROP (GALANTHUS L.) HERBAL PHARMACEUTICAL SUBSTANCES BY ULTRAVIOLET-SPECTROPHOTOMETRY
}

\author{
DMITRY OLEGOVICH BOKOV ${ }^{1,2 *}$ \\ ${ }^{1}$ Department of Pharmaceutical and Natural Sciences, Sechenov First Moscow State Medical University, 8, Trubetskaya St., Moscow, \\ 119991, Russia. ${ }^{2}$ Laboratory of food chemistry, Federal Research Center for Nutrition, Biotechnology and Food Safety, $2 / 14$, Ustyinsky pr., \\ Moscow, 109240, Russia. Email: fmmsu@mail.ru \\ Received: 24 April 2018, Revised and Accepted: 14 June 2018
}

ABSTRACT

Objective: The objective of the present study is to evaluate the electronic ultraviolet (UV) absorption spectra of herbal pharmaceutical substances (70\% ethanol extracts) prepared from medicinal plant material of two snowdrop species - Galanthus woronowii Losinsk and Galanthus nivalis L.

Methods: The groups of biologically active substances were investigated by UV-spectrophotometry including special sample preparation for flavonoids and alkaloids.

Results: In the present study, data were obtained characterizing the composition of the main biologically active compounds of the genus Galanthus L., received its "spectrophotometric profiles." On the basis of spectral characteristics of G. woronowii and G. nivalis flavonoids and alkaloids, we confirm feasibility for using galantamine, lycorine, quercetin, and its glycosides (hyperoside et al.) state standard samples in standardization analysis.

Conclusion: It is shown that this method can be used to assess the quality of herbal pharmaceutical substances, serve to prove their identity in pharmacopoeial monograph.

Keywords: Ultraviolet-spectrophotometry, Galanthus, Herbal pharmaceutical substances, Amaryllidaceae alkaloids, Flavonoids.

(C) 2018 The Authors. Published by Innovare Academic Sciences Pvt Ltd. This is an open access article under the CC BY license (http://creativecommons. org/licenses/by/4. 0/) DOI: http://dx.doi.org/10.22159/ajpcr.2018.v11i10.26867

\section{INTRODUCTION}

Recently, there is a growing interest in relation to the homeopathic method of treatment, as well as a significant increase in the volume of registration of new homeopathic drugs (HDs) in Russian Federation. These trends have led to a new chapter in the domestic pharmacy homeopathic pharmacy that study HD used in health care according to the principles of homeopathy. The main priorities of homeopathic pharmacy are development and improvement of the regulatory framework document, which regulates the production and quality control HD, increase HD range of domestic, as well as development and improvement of modern methods of analysis, quality control, and standardization $[1,2]$.

HD quality assurance largely dependents on the use of standard homeopathic substances produced according to the good manufacturing practice. Homeopathic mother tinctures (HMT) are the feedstock for most HD. HMT is liquid hydroalcoholic extracts from raw materials of plant (or animal) origin. Most often HMT is produced by maceration (also percolation is possible). Quality control of HMT is to identify biologically active substances (BAS), the definition of authenticity, as well as other indicators that are defined privately normative documentation $[3,4]$.

Our research was conducted at the Pharmaceutical and Natural Sciences Department (Pharmacy Institute) at the Sechenov First Moscow State Medical University. There normative documents for standardization of HMT and preparations developed purposefully over the last decades.

HDs of snowdrops are widely used abroad in the treatment of headaches, myocarditis, and diseases of the cardiovascular system. The raw material of common snowdrop (Galanthus nivalis L.) is used for the production of HMT [5], Voronov's snowdrop (Galanthus woronowii
Losinsk) is also allowed for this purpose in Russia [6]. Appearance of snowdrops is shown at Fig. 1.

The relevance of this study is due primarily to the lack of national standardization methods of homeopathic pharmacy for HMT snowdrops derived from fresh flowering snowdrop plants of G. woronowii and G. nivalis (Amaryllidaceae J.St.-Hill.). Amaryllidaceae plants are often used in medicine for its unique properties [7-9]. In particular, the method of HMT preparation is unknown, qualitative reactions for determining the presence of Amaryllidaceae alkaloids [10,11] and flavonoids [12] (and other BAS groups) in HMT are not defined and there are no pharmacopoeial monographs for G. woronowii and G. nivalis homeopathic crude herbal drug (CHD) and HMT.

In pharmacognostic practice, ultraviolet (UV)-spectrophotometry method is most often used for the analysis of CHD and preparations based on it when it is necessary to study the total content of BAS. It should be noted that the UV-spectrophotometric analysis is quite easy to conduct, accurate, and economically cost effective, which accounts for its successful application to standardize [13]. It is applicable for the analysis of a number of com pounds having chromophoric groups (flavonoids, alkaloids, and other compounds). A spectrophotometric procedure was developed for the determination of total alkaloids content in Tinospora cordifolia M. with bromocresol green [14].

Flavonoid compounds contained in plant and pharmaceutical objects consist principally of a plurality of glycosides mixtures, which belong to different classes of polyphenols. It greatly complicates the selection methods of their determination and requires extra system studies of chemical composition. So far, a detailed spectrophotometric characterization of polyphenolic compounds found in snowdrops is not carried out [15]. Total flavonoids content is often determined by differential spectrophotometry with aluminum chloride in CHD [16-18]. 

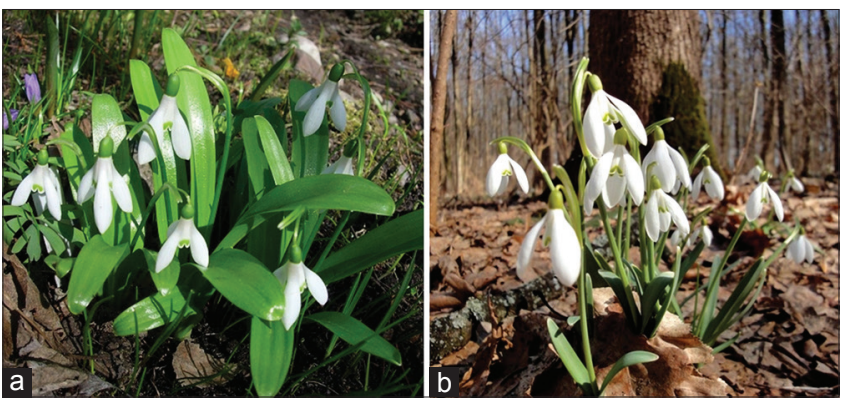

Fig. 1: (a) Voronov's snowdrop (Galanthus woronowii Losinsk); (b) common snowdrop (Galanthus nivalis $\mathrm{L}$.)

\section{METHODS}

\section{Chemicals}

The following standard samples were used in the work: the standard sample of quercetin, CAS №. 117-39-5 (LLC "Phytopanacea” Russia, a series of 130,213 , purity $\geq 99.5 \%$ ), the standard samples and galantamine CAS №. 357-70-0 Lycorine CAS №. 476-28-8 (Nanjing Spring and Autumn Biological Engineering Co., Ltd, Jiang Su, China, high-performance liquid chromatography $\geq 98 \%$ ).

\section{Plant material}

The objects of study were samples of Voronov's snowdrop ( $G$. woronowii Losinsk) and common snowdrop (G. nivalis L.) harvested at the flowering stage at the Botanical Garden of Sechenov First Moscow State Medical University in Moscow (April-March 2016-2018).

\section{HMT preparation}

Pharmacopoeial HMTs were prepared according to the method 3a "HMT" [19] from the whole flowering plants (Planta tota). The choice of this method is based, primarily, due to the fact that plants of the Galanthus $\mathrm{L}$. genus contain a substantial amount of mucus. It has also been prepared a series of infusions from underground parts (only bulbs with roots), without the aerial part for analytical purposes. The resulting infusion of the whole plant is a clear aqueous-alcohol extract of yellow-brown color with a greenish hue and a specific smell. HMT obtained from underground parts - transparent brownish liquid also has a specific smell.

\section{Determination of BAS}

An identification tests were carried out with HMT to determine the major BAS groups included in the GPh XI edition $[20,21]$ and also that is widely used in scientific research.

To determine the Amaryllidaceae alkaloids, we use direct spectrophotometry - for HMT derived from underground parts; extraction-spectrophotometric method - for HMT obtained from whole plants. Scheme extraction-spectrometric method is as follows:

- Preparation of "Tropeolin 000-II - alkaloid". To aliquot of HMT, we added $1 \%$ hydrochloric acid solution that was stirred and placed in a separatory funnel, then we added $1 \%$ solution of tropeolin 000-II. The ion associate structure of the Tropeolin 000-II and galantamine can be represented as follows:

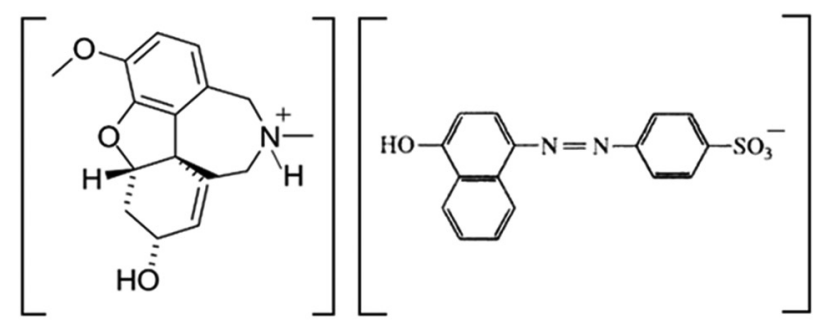

- Extraction of the resulting complex in the organic phase (extraction with chloroform three times) and measuring the optical density in obtained chloroform extract (orange-colored).
An acid hydrolysis of flavonoid glycosides in HMT was carried out for the analysis of flavonoids. $5 \mathrm{ml}$ of HMT was evaporated on a water bath; the residue was dried in an oven at $105^{\circ} \mathrm{C}$ for $10 \mathrm{~min}$, then dissolved in $15 \mathrm{ml}$ of $10 \%$ sulfuric acid. Hydrolysis was carried out in a flask with ground joint volume of $100 \mathrm{ml}$, connected to a reflux condenser under heating in a boiling water bath for $2.5 \mathrm{~h}$. Under these conditions, the hydrolysis of 3-0-glycosides advantageously takes place as 7-0-glucosides are more stable and are hydrolyzed under more severe conditions. Then, the flask was cooled to room temperature under a stream of cold water; the contents were filtered through filter paper. The precipitate remaining on the filter was washed with purified water and dissolved in $25 \mathrm{ml}$ of hot $96 \%$ ethanol with the addition of $3 \mathrm{ml}$ of a $2 \%$ solution of aluminum chloride, and the resulting solution was subjected to spectrophotometric analysis.

A study of the HMT electronic spectra was performed by the instrument "Cary 50 Scan" company Agilent Technologies (formerly - Varian, USA), followed by computer processing of the results of the program "Cary WinUV Analysis Pack ver. 3.1" for "Windows."

\section{RESULTS AND DISCUSSIONS}

As a result of identification tests in two snowdrops species HMT, we confirmed the presence of two BAS groups - flavonoids and alkaloids in HMT made from whole plants. As the biosynthesis of flavonoids and other polyphenol compounds nature occurs in aerial parts of plants, tinctures derived from underground parts lack of this BAS group. The results of qualitative reactions are shown in Table 1.

The analysis of the electronic spectra of standard samples of galantamine, lycorine, and snowdrops HMT (Fig. 2) obtained from the underground parts established the presence of two peaks approximately the same average width at $210 \mathrm{~nm}$ and $285 \mathrm{~nm}$.

The first highest peak at $210 \pm 3 \mathrm{~nm}(2 \mathrm{~b})$ is characterized by a smooth left slope and a small bend on the right side, the second maximum at $285 \pm 3 \mathrm{~nm}$ is much smaller than the first. In general, the absorption maxima of the two compounds with the following structure coincide:
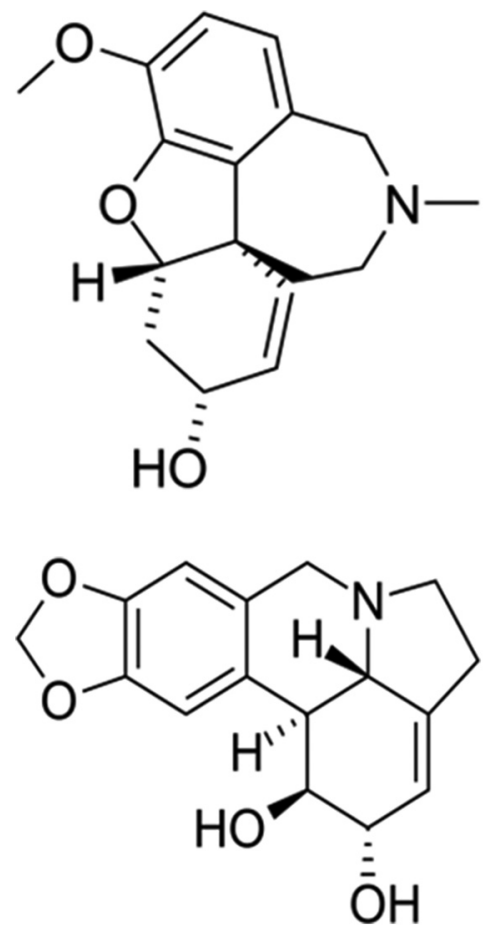

Galantamine, $\mathrm{C}_{17} \mathrm{H}_{21} \mathrm{NO}_{3} \quad$ Lycorine, $\mathrm{C}_{16} \mathrm{H}_{17} \mathrm{NO}_{4}$ 
Table 1: The results of identification tests for BAS groups in HMT of G. woronowii and G. nivalis

\begin{tabular}{|c|c|c|c|c|c|}
\hline \multirow[t]{2}{*}{ Group of BAS } & \multirow[t]{2}{*}{ Reagent } & \multicolumn{2}{|c|}{ HMT of $G$. woronowii } & \multicolumn{2}{|c|}{ HMT of G. nivalis } \\
\hline & & Whole plant & Underground parts & Whole plant & Underground parts \\
\hline \multirow[t]{6}{*}{ Amaryllidaceae alkaloids } & $\begin{array}{l}\text { A solution of iodine in potassium } \\
\text { iodide (Wagner-Bouchard reagent) }\end{array}$ & + & + & + & + \\
\hline & $\begin{array}{l}\text { A solution of potassium iodide, } \\
\text { bismuth iodide (Dragendorff reagent) }\end{array}$ & + & + & + & + \\
\hline & $\begin{array}{l}\text { A solution of silicotungstic } \\
\text { acid (reagent Bertrand) }\end{array}$ & + & + & + & + \\
\hline & $\begin{array}{l}\text { A solution of phosphomolybdic } \\
\text { acid (reagent sonnenstein) }\end{array}$ & + & + & + & + \\
\hline & A solution of picric acid & + & + & + & + \\
\hline & A solution of tannin & + & + & + & + \\
\hline \multirow[t]{3}{*}{ Flavonoids } & $2 \%$ alcohol solution of $\mathrm{AlCl}_{3}$ & + & - & + & - \\
\hline & $\begin{array}{l}\text { Boric acid and citric acid anhydrous } \\
\text { ethyl alcohol (Wilson reagent) }\end{array}$ & + & - & + & - \\
\hline & $\begin{array}{l}\text { Shinoda's test (Mg metal. in the } \\
\text { presence of } \mathrm{HCl} \text { conc.) }\end{array}$ & + & - & + & - \\
\hline
\end{tabular}

HMT: Homeopathic mother tinctures, BAS: Biologically active substances, G. woronowii: Galanthus woronowii, G. nivalis: Galanthus nivalis

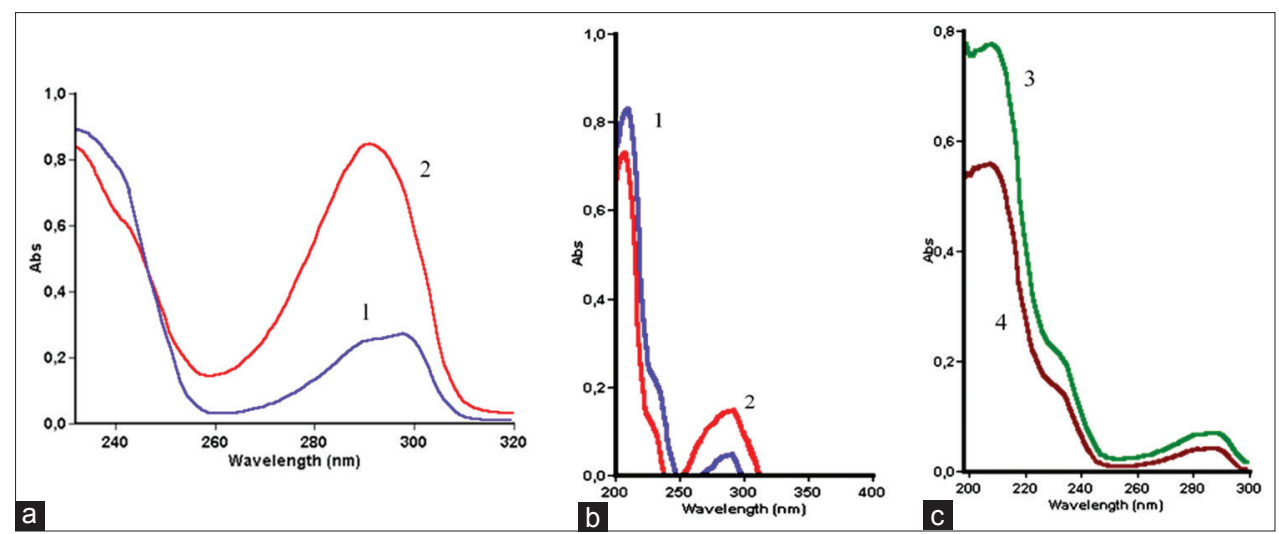

Fig. 2: The absorption spectra of standard samples of galanthamine (1) lycorine (2) 235-320 nm (a) and 200-400 nm (b) and homeopathic mother tinctures (c) of Galanthus woronowii (3) and the Galanthus nivalis (4) 200-300 nm

It can be assumed that the detected shape of the absorption spectral curves of lycorine and galantamine, related to the isoquinoline derivatives, is the consequence of the presence in their molecules of cyclic chromophores that are associated with the group $=\mathrm{N}-\mathrm{C}$.

Electronic absorption spectra of snowdrops HMT, derived from underground parts, have similar absorption maxima to analyzed standard samples (Fig. 2c).

HMT absorption spectra obtained from whole plants in the wavelength range have a plurality of absorption maxima in the range of 200$320 \mathrm{~nm}$, due to the presence of a number of polyphenolic compounds and other impurities. Absorptions in the short-wave part of the spectrum are difficult to interpret because of the large number of absorption peaks overlapping each other to a large extent. This makes it difficult and makes it impossible to analyze the Amaryllidaceae alkaloids, so we used extraction-spectrophotometric method described in the standard documentation and the scientific literature. Complex formation galantamine with tropeolin 000-II is used for the quantitative determination of galantamine in the substance of galantamine hydrobromide [22] and in the pharmacopoeial monograph for Ungernia victoris Vved. CHD [23]. This technique allows to analyze HMT snowdrops derived from whole plants as selective extraction complex "tropeolin-alkaloid" allows you to get rid of unwanted impurities. Spectra of galantamine, lycorine, and HMT of G. woronowii and G. nivalis are shown in Fig. 3a. Absorption maximum at $480 \pm 3 \mathrm{~nm}$ is characteristic for galantamine and for lycorine. The total amount of these alkaloids is contained in HMTs. It should be considered in the standardization procedure of CHD and HMT. It is necessary to introduce new index: alkaloid total content in terms of galantamine or lycorine.

Taking into account the fact that HMT derived from whole plants contain many polyphenolic compounds, we have attempted to characterize some interpreted absorption maxima. One of these peaks observed at $330 \pm 5 \mathrm{~nm}$, and there is a slight bend at $400 \mathrm{~nm}$ (the peak is not fixed); this characteristic can be associated with hydroxycinnamic acids [24]. Electronic absorption spectra of $G$. woronowii and G. nivalis HMT presented in Fig. 3b. It should be noted that the spectra are given in Fig. 3b may serve as a characteristic of officinal HMTs authenticity.

Quercetin was selected as standard sample according to the obtained intermediate experimental and published data. After the hydrolysis of HMT and subsequent treatment with $2 \%$ solution of aluminum chloride, we observed the absorption maximum at $430 \pm 5 \mathrm{~nm}$. Obviously, during the hydrolysis of quercetin glycosides prevailing in G. woronowii and G. nivalis HMT, broken, releasing the free quercetin. Quercetin with $2 \%$ ethanolic solution of aluminum chloride forms a colored compound having a yellow-green fluorescence at a wavelength of about $430 \mathrm{~nm}$ (bathochromic shift) (Fig. 4).

Structural formulas of quercetin and its most frequently occurring glycosides are shown below: 


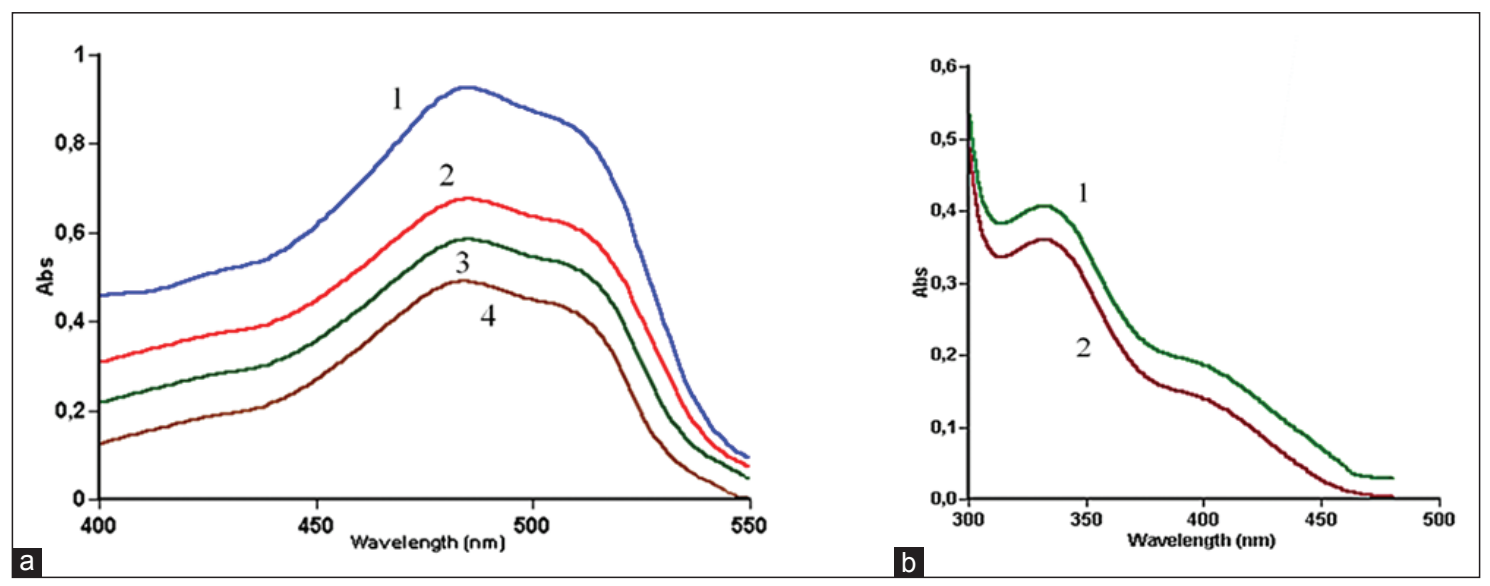

Fig. 3: The absorption spectra of the chloroform extract containing complex of tropeolin II-000 with standard samples of galanthamine (1), lycorine (2), homeopathic mother tinctures (HMT) of Galanthus woronowii (3) at 400-550 nm (a) and Galanthus nivalis (4) at 400-550 nm (a); individual absorption spectra HMT of G. woronowii (1) and G. nivalis (2), at 300-500 nm (b)

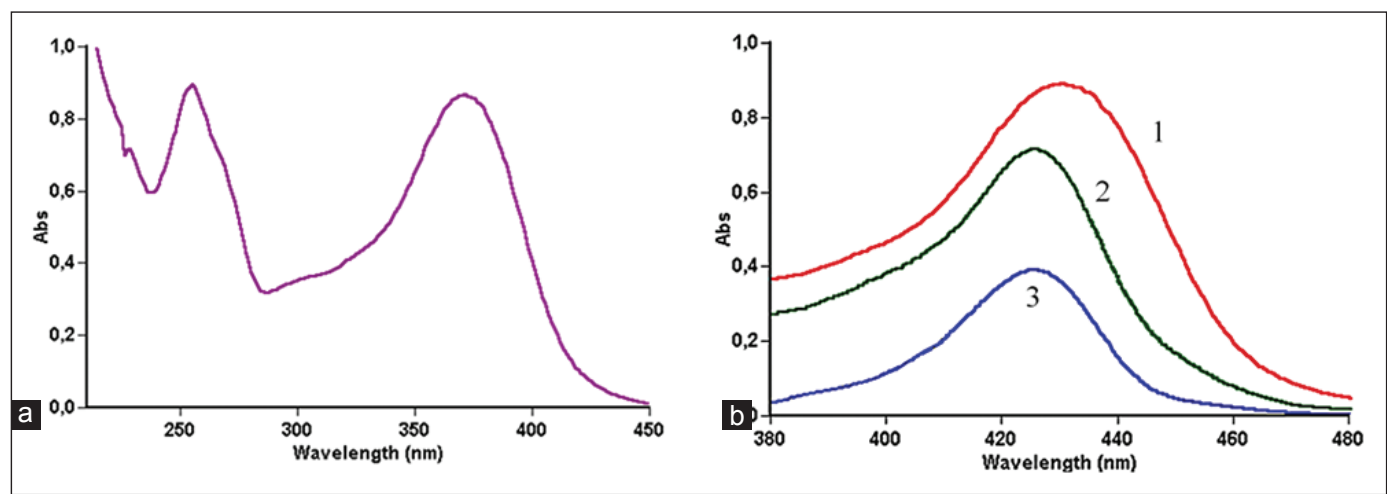

Fig. 4: The absorption spectrum of the quercetin standard sample at 200-450 $\mathrm{nm}$ (a); the absorption spectra of quercetin (1) and hydrolysis products homeopathic mother tinctures of Galanthus woronowii (2) and Galanthus nivalis (3) with a $2 \%$ solution of aluminum chloride at 380-480 $\mathrm{nm}(\mathrm{b})$<smiles></smiles><smiles>O=c1c(OCC2(O)C(O)C(O)C(O)C(O)C2O)c(-c2ccc(O)c(O)c2)oc2cc(O)cc(O)c12</smiles>

Quercetin -3,5,7,3 ', 4'-pentoxide flavon Quercetin glycoside quercetin 3-O- $\beta$-D-galactoside (hyperoside)

Flavonoid compounds are secondary metabolite in plants; they show a wide spectrum of biological and pharmacological functions. Among the all flavonoids, quercetin gained special attention for its potential therapeutic activities (anti-inflammatory, antioxidant, antitoxic, anticancer, immunomodulatory, and others) [25]. Taking into account this aspect, we can assume that the snowdrops drugs will be in demand on the pharmaceutical market.

\section{CONCLUSION}

Qualitative analysis for the main BAS groups of $G$. woronowii and G. nivalis herbal pharmaceutical substances - HMT was conducted. We defined by UV-spectrophotometry that they are Amaryllidaceae alkaloids and flavonoids. On the basis of spectral characteristics of G. woronowii and G. nivalis HMT flavonoids and alkaloids, we confirm method feasibility for using galantamine, lycorine, quercetin, and its glycosides (hyperoside et al.) state standard samples in standardization analysis. Extraction-photometric method can be applied successfully for standardization and the determination of galantamine and lycorine in G. woronowii and G. nivalis HMT and CHD. The authenticity of HMT of $G$. woronowii and G. nivalis can be confirmed by electronic absorption spectrum with a maximum at $330 \pm 5 \mathrm{~nm}$ and bend at $400 \mathrm{~nm}$.

\section{ACKNOWLEDGMENT}

I would like to thank professor, corresponding member of Russian Academy of Sciences Irina Aleksandrovna Samylina for her useful communications and constant help.

\section{AUTHOR' CONTRIBUTIONS}

I declare that this work was done by the author named in this article.

\section{CONFLICTS OF INTEREST}

None.

\section{REFERENCES}

1. Bandaranayake WM. Quality control, screening, toxicity, and regulation of herbal drugs. In: Modern Phytomedicine: Turning Medicinal Plants into Drugs. Weinheim: Wiley-VCH Verlag GmbH \& Co.; 2006. p. $25-57$

2. Samylina IA, Kostennikova ZP, Tereshina NS, Tsukanov YuV. Scientific approaches to developing general pharmacopoeial monograph on homeopathic formulations. Pharmacy 2010;3:53-6.

3. Shikh EV, Bulayev VM, Demidova OA, Bunyatyan ND. On 
homeopathic medicines allowed for medical use in the Russian federation. Sci Cent Expert Med Appl Prod Bull 2014;4:50-4.

4. Kopytko YF, Sokolskaya TA, Dargaeva TD. Regulation of quality matrix homeopathic tinctures and row materials for their manufacture in Russia and abroad. Tradit Med 2010;23:53-6.

5. Homoepathic Pharmacopoeia of the United States. Revision Service. T. 1: December 1988-December 1990; T. 2: December 1989-June 1990.

6. The Order of the Ministry of Health of the Russian Federation № 335 from 11.29.95 "On the use of the Homeopathic Method in Practical Public Health. Available from: http://www.andriana.ru/article3.html. [Last accessed on 2018 Apr 20].

7. Taha KF, El-Hawary SS, El-Hefnawy HM, Mabrouk MI, Sanad RA, El Harriry MY. Formulation and assessment of a herbal hair cream against certain dermatophytes. Int J Pharm Pharm Sci 2016;8:167-73.

8. Roy S, Awasthi H. Herbal medicines as neuroprotective agent: A mechanistic approach. Int J Pharm Pharm Sci 2017;9:1-7.

9. Divya BJ, Suman B, Venkataswamy M, Thyagaraju K. A study on phytochemicals, functional groups and mineral composition of Allium sativum (garlic) cloves. Int J Curr Pharm Res 2017;9:42-5.

10. Berkov S, Codina C, Viladomat F, Bastida J. Alkaloids from Galanthus nivalis. Phytochemistry 2007;68:1791-8.

11. Sarikaya BB, Kaya GI, Onur MA, Bastida J, Somer NU. Phytochemical investigation of Galanthus woronowii. Biochem Syst Ecol 2013;51:276-9.

12. Bokov DO, Samylina IA. Identification of flavonoids in homeopathic mother tinctures of Galanthus woronowii Losinsk. and Galanthus nivalis L. By ultra-performance liquid chromatography with photodiode arrays and tandem quadrupole mass-selective detectors. Pharm Chem J 2016;50:458-64

13. Koldaev VM. Optical absorption spectrophotometry in pharmacy. Pac Med J 2014;1:19-23.

14. Patel RK, Patel JB, Trivedi PD. Spectrophotometric method for the estimation of total alkaloids in the Tinospora cordifolia M. And its herbal formulations. Int J Pharm Pharm Sci 2015;7:249-51.
15. Bokov DO, Samylina IA. Homeopathic remedies of snowdrops: Key aspects for standardization procedures. Proceedings of the V international scientific-practical conference fundamental science and technology-promising developments (24-25 February 2015). Pharm Sci (North Charleston, USA) 2015;2:184-6.

16. Kim JH, Kim MY. Phytochemical and antioxidant characterization of thinned immature Citrus unshiu fruits. Int J Pharm Pharm Sci 2017:9:293-7.

17. Sreedevi P, Vijayalakshmi K, Venkateswari R. Phytochemical evaluation of Punicagranatum L. Leaf extract. Int J Curr Pharm Res 2013;9:14-8.

18. Jain KL, Choudhury PK, Sharma M. Total flavonoid quantification and to study antibacterial potency of extracts of Butea monosperma flowers, Nigella sativa seeds and Vitex agnus-castus leaves. Int J Curr Pharm Res 2017;9:71-4

19. Homeopathic Mother Tinctures, "Tincturae Homoeopathicae Maternae". The Ministry of Health of the Russian Federation. Available from: http://www.rosminzdrav.ru. [Last accessed on 2018 Apr 20]

20. The State Pharmacopoeia of the USSR. General Methods of Analysis. $11^{\text {th }}$ ed. Moscow: Medicine; 1987.

21. The State Pharmacopoeia of the USSR. General Methods of Analysis. $11^{\text {th }}$ ed. In: Medicinal Herbs. Moscow: Medicine; 1989.

22. Medicine. The State Pharmacopoeia of the USSR. $10^{\text {th }}$ edition. Moscow: Medicine; 1968.

23. Pharmacopoeia monograph 42-1520-80 Leaves of Ungernia victoris Vved. ("Folia Ungernia victoris"). Medicinal plants of the State Pharmacopoeia. In: Samylina IA, Severtsev VA, editors. Pharmacognosy. Moscow: ANMI; 2003. p. 456-8.

24. Bokov DO, Samylina IA, Malinkin AD, Bessonov VV. Hydroxycinnamic and organic acids of snowdrops (Galanthus L.) J Appl Pharm Sci 2017;2:36-40

25. Nathiya S, Durga M, Devasena T. Quercetin, encapsulated quercetin and its application-a review. Int J Pharm Pharm Sci 2014;6:20-6. 